6H-SiC Photoconductive

Switches Triggered at Below Bandgap Wavelengths

J. S. Sullivan, J. R. Stanley

February 27, 2007

IEEE Transactions on Dielectrics and Electrical Insulation 
This document was prepared as an account of work sponsored by an agency of the United States Government. Neither the United States Government nor the University of California nor any of their employees, makes any warranty, express or implied, or assumes any legal liability or responsibility for the accuracy, completeness, or usefulness of any information, apparatus, product, or process disclosed, or represents that its use would not infringe privately owned rights. Reference herein to any specific commercial product, process, or service by trade name, trademark, manufacturer, or otherwise, does not necessarily constitute or imply its endorsement, recommendation, or favoring by the United States Government or the University of California. The views and opinions of authors expressed herein do not necessarily state or reflect those of the United States Government or the University of California, and shall not be used for advertising or product endorsement purposes. 


\title{
6H-SiC Photoconductive Switches Triggered at Below Bandgap Wavelengths
}

\author{
J.S.Sullivan and J.R. Stanley \\ University of California \\ Lawrence Livermore National Laboratory \\ Livermore,CA 94550, USA
}

\begin{abstract}
Semi-insulating silicon carbide ( $\mathrm{SiC}$ ) is an attractive material for application as high voltage, photoconductive semiconductor switches (PCSS) due to its large bandgap, high critical electric field strength, high electron saturation velocity and high thermal conductivity. The critical field strength of $300 \mathrm{MV} / \mathrm{m}$ for $6 \mathrm{H}-\mathrm{SiC}$ makes it particularly attractive for compact, high voltage, fast switching applications. To realize the benefits of the high bulk electric field strength of $\mathrm{SiC}$ and diffuse switch current, carriers must be excited throughout the bulk of the photo switch. Photoconducting switches with opposing electrodes were fabricated on "a" plane, vanadium compensated, semiinsulating, $6 \mathrm{H}-\mathrm{SiC}$ substrates. The PCSS devices were switched by optically exciting deep extrinsic levels lying within the $6 \mathrm{H}-\mathrm{SiC}$ bandgap. The $\mathrm{SiC}$ photoswitches were tested up to a bias voltage of $11000 \mathrm{~V}$ with a corresponding peak current of $150 \mathrm{~A}$. The $6 \mathrm{H}-\mathrm{SiC}$ substrates withstood average electric fields up to $27 \mathrm{MV} / \mathrm{m}$. Minimum PCCS dynamic resistances of 2 and $10 \Omega$ were obtained with $13 \mathrm{~mJ}$ optical pulses at 532 and $1064 \mathrm{~nm}$ wavelengths, respectively.
\end{abstract}

Index Terms - Photoconducting devices, photoconductivity, light triggered switches, semiconductor switches, silicon carbide.

\section{INTRODUCTION}

THE $6 \mathrm{H}-\mathrm{SiC}$ polytype has a wide band-gap $(3 \mathrm{eV})$, high critical field strength $(300-400 \mathrm{MV} / \mathrm{m})$, high-saturated electron velocity $\left(2.0 \times 10^{7} \mathrm{~cm} / \mathrm{s}\right)$ and high thermal conductivity $\left(4.9 \mathrm{~W} / \mathrm{cm}^{\circ} \mathrm{C}\right)$. These material properties make semi-insulating $6 \mathrm{H}-\mathrm{SiC}$ an attractive semiconductor material for the Photoconductive Semiconductor Switch (PCSS) application. Previous SiC PCSS work [1-3] used high resistivity, low impurity $\mathrm{SiC}$ polytypes and focused on lateral geometry surface switches that used above band-gap wavelengths of light to trigger the switches. The performance and switch life of lateral geometry PCSS are limited by surface flashover, surface carrier mobility and high current density. PCSS with opposing electrical contacts deposited on vanadium compensated, semi-insulating, $6 \mathrm{H}-\mathrm{SiC}$ substrates can be triggered using below band-gap light to excite carriers from extrinsic levels throughout the bulk of the material. This results in diffuse photocurrent and switch hold off voltages determined by the bulk breakdown field strength of $6 \mathrm{H}-\mathrm{SiC}$. The bulk switching capability and semi-insulating nature of $6 \mathrm{H}-\mathrm{SiC}$ are enabled by the addition of the dopant vanadium. Vanadium is an amphoteric impurity that can act as a deep acceptor, or a deep donor. The local Fermi level in the $6 \mathrm{H}-\mathrm{SiC}$ is determined by the relative densities of the impurities, nitrogen, boron and vanadium present in the material.

Manuscript received on 15 November 2006, in final form 22 February 2007.
Vanadium acts as a deep acceptor when the nitrogen donor impurity density sufficiently exceeds the boron acceptor impurity density, which is the case for the $6 \mathrm{H}-\mathrm{SiC}$ material we have tested.

Another feature of this material is that the Fermi level will be pinned close to the vanadium acceptor level. The deep $(0.7$ $\mathrm{eV}$ below conduction band) vanadium acceptor levels accept electrons donated from the shallow $(0.08 \mathrm{eV}$ below conduction band) nitrogen donor levels resulting in semiinsulating $6 \mathrm{H}-\mathrm{SiC}$ material. The electrons residing in the extrinsic vanadium acceptor levels can be excited into the conduction band by photons with energies exceeding $0.7 \mathrm{eV}$. Figure 1 [4] depicts the extrinsic levels in the $6 \mathrm{H}-\mathrm{SiC}$ bandgap. The Boron acceptor levels and defect levels are also shown in Figure 1. The defect levels include silicon vacancies and the UD-1 defect [4].

\section{PCSS TEST APPARATUS AND PROCEDURE}

Five PCSS devices were fabricated from samples of 400 $\mu \mathrm{m}$ thick, $1.2 \mathrm{~cm}$ per side, square substrates of "a" plane, vanadium compensated, semi-insulating $6 \mathrm{H}-\mathrm{SiC}$. The four facets of the substrate were cleaved/polished to enhance optical coupling into the bulk of the substrate. The contacts consist of a $0.8 \mathrm{~cm}$ diameter, circular metalization centered on opposing sides of the substrate. The metalization formed an ohmic contact and consisted of layers of nickel, titanium, 
platinum and gold. The metal deposition and anneal were performed at SemiSouth Laboratories Inc. in Starksville, MS. Indium coated copper electrodes were brazed to the substrate metalizations to facilitate electrical connection. The finished $6 \mathrm{H}-\mathrm{SiC}$ PCSS assembly is shown in Figure 2. The $6 \mathrm{H}-\mathrm{SiC}$ PCSS assembly was prepared for photoconductivity measurements by cleaning the PCSS facets to remove any

\section{Conduction Band}

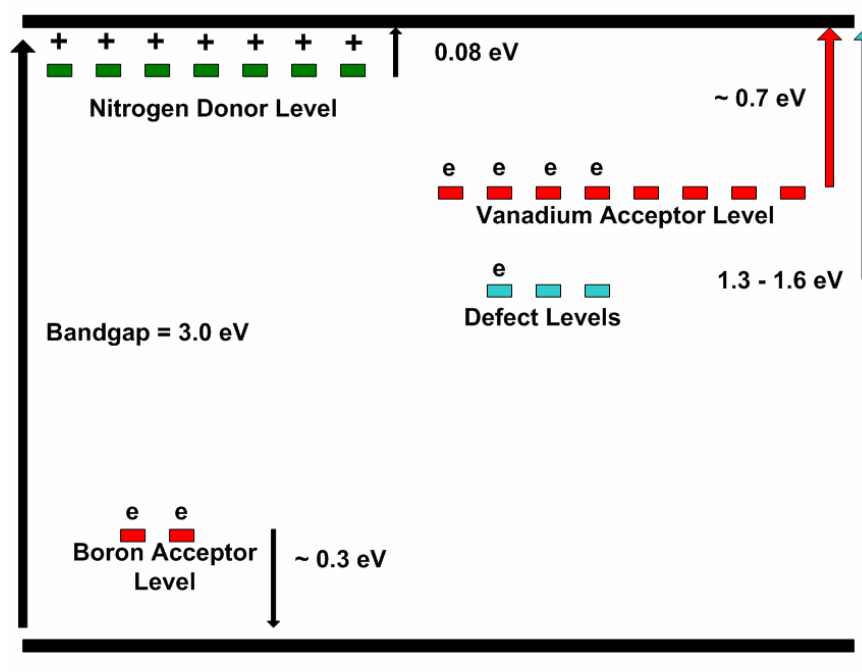

\section{Valence Band}

Figure 1. Vanadium acceptor and nitrogen donor levels in vanadium compensated $6 \mathrm{H}-\mathrm{SiC}$.

organic residue. All four PCSS facets were inspected by microscope and given identification marks prior to photoconductivity tests. Photoconductivity tests were performed using $1064 \mathrm{~nm}$ and frequency doubled $532 \mathrm{~nm}$ wavelength light from a Q-switched Nd:YAG laser with an 8 ns at full wave half maximum (FWHM) output pulse. The optical pulse was focused and aligned to obtain as uniform as possible light pulse over a rectangular area measuring $1 \mathrm{~cm}$ wide by $400 \mu \mathrm{m}$ high. The optical pulse was then centered on the PCSS facet. Photoconductivity tests were performed using optical pulse energies ranging from 1 to $14 \mathrm{~mJ}$. The optical energy was increased in 1-mJ increments using a waveplatepolarizer pair. PCSS facets were inspected for damage by microscope between optical pulse energy increments.

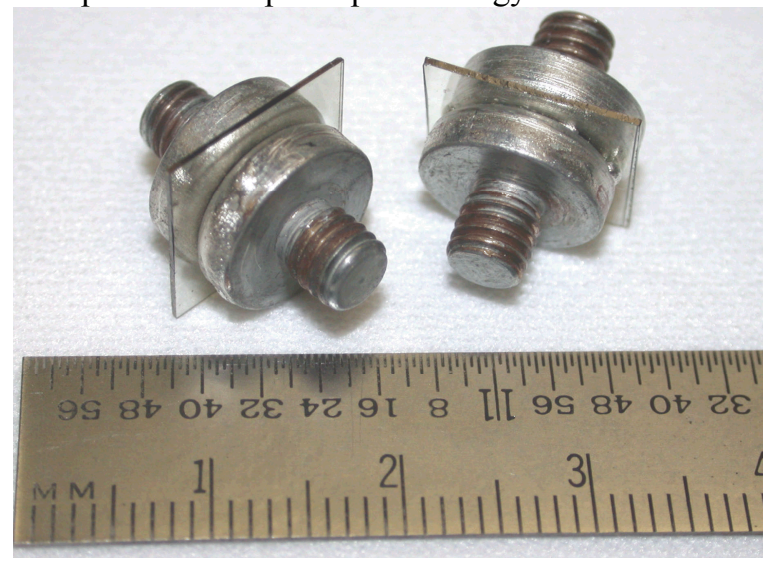

Figure 2. Vanadium compensated, semi-insulating, 6H-SiC PCSS assembly.
The photoconductivity of the PCSS was measured using the circuit shown in Figure 3. The test circuit was constructed on 0.125 inch thick, $56 \mathrm{~g}(2 \mathrm{oz}) \mathrm{Cu}$ printed circuit board. The load resistor and storage capacitor were positioned on opposite sides of the 2.5 inch long, 1.9 inch wide circuit board and were constructed from a series and parallel combination of components to minimize circuit inductance. The total circuit inductance was estimated to be $12 \mathrm{nH}$. The $1.5 \mu \mathrm{F}$ capacitor of the test circuit was pulse charged to bias voltages ranging from $250 \mathrm{~V}$ to $4.25 \mathrm{kV}$ in $30 \mu \mathrm{s}$. The PCSS was optically triggered after a $30 \mu \mathrm{s}$ flat top interval of the pulse bias voltage. The voltage across the PCSS was measured differentially using a pair of calibrated, fast $(250 \mathrm{MHz}$ bandwidth), high voltage $(5 \mathrm{kV})$ probes. The tips of the voltage probes were inserted in pin sockets soldered onto the switch electrode connections. The load voltage was measured using a fast (35 ps rise time), low impedance (250 $\Omega$ ) high voltage probe. The PCSS current was obtained by dividing the load voltage by the load resistance. Dynamic switch resistance is another important switching parameter. Dynamic switch resistance is a measure of how well the applied optical pulse has reduced the bulk resistivity of the $6 \mathrm{H}-\mathrm{SiC}$ substrate. The dynamic switch resistance is calculated by dividing the switch voltage by the load current.

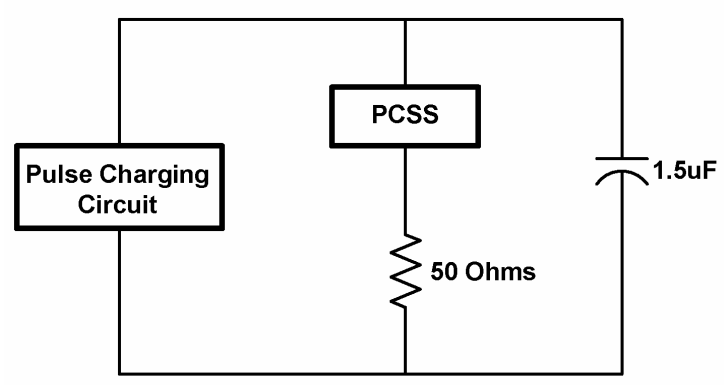

Figure 3. Simple schematic for PCSS photoconductivity test circuit.

\section{EXPERIMENTAL RESULTS}

\subsection{Minimum Dynamic Resistance}

The PCSS voltage and current for a $4.25 \mathrm{kV}$ charge voltage and optical pulse energy of $13 \mathrm{~mJ}$ at $1064 \mathrm{~nm}$ are shown in Figure 4. The PCSS voltage starts at $4.25 \mathrm{kV}$ and collapses to approximately 750 Volts in $10 \mathrm{~ns}$, while the PCSS current increases from zero to $70 \mathrm{~A}$. The PCSS current pulse is similar in temporal profile to the $1064 \mathrm{~nm}$ optical trigger pulse. However, the photocurrent has a 16 ns FWHM pulsewidth indicating a carrier recombination time of a few nanoseconds, or, less. The minimum dynamic PCSS resistance for the pulse shown in Figure 4 is $11 \Omega$. The minimum dynamic PCSS resistance is approximately constant for fixed optical pulse energy, regardless of the charge voltage. Switch minimum dynamic PCSS resistance as a function of optical pulse energy for 1064 and $532 \mathrm{~nm}$ wavelengths is shown in Figure 5. The switch minimum dynamic PCSS resistance decreases rapidly with optical pulse energy for both optical wavelengths. The PCSS attains a 
lower minimum dynamic resistance at all optical energies for excitation with $532 \mathrm{~nm}$ light compared to excitation with $1064 \mathrm{~nm}$ light. This is a result of charge carriers being excited from additional extrinsic levels by the $532 \mathrm{~nm}$ wavelength.

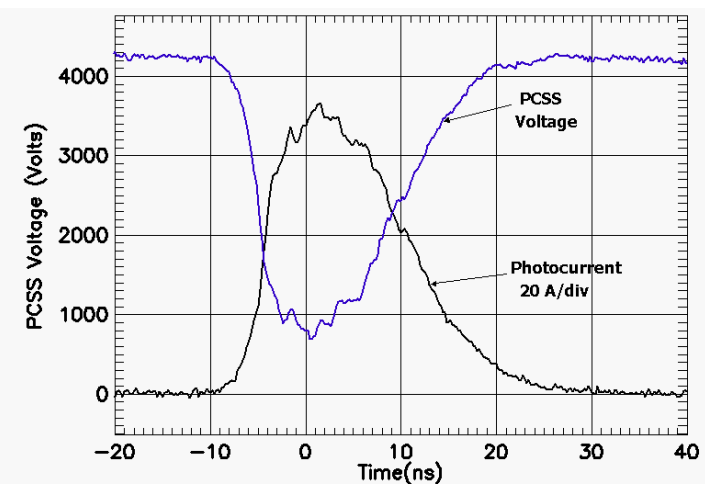

Figure 4. PCSS voltage and current for bias voltage of $4.25 \mathrm{kV}$ and $13 \mathrm{~mJ}$ optical trigger at $1064 \mathrm{~nm}$.

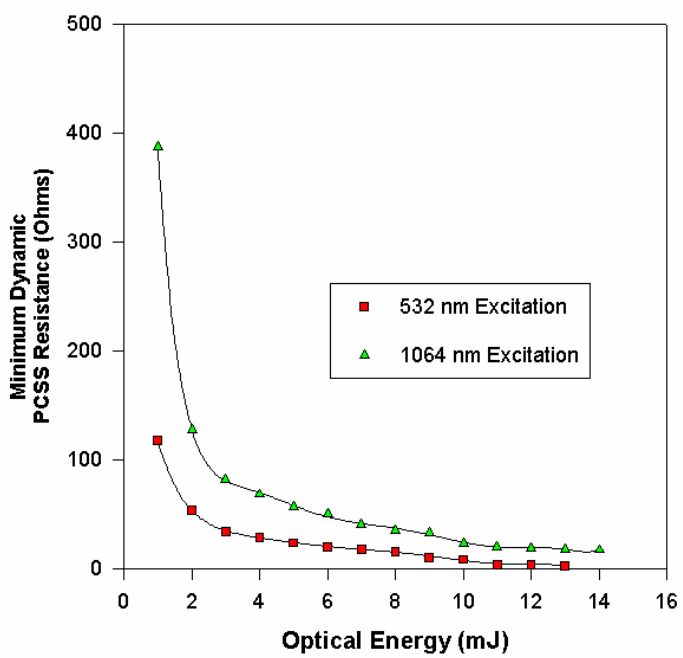

Figure 5. Minimum dynamic PCSS resistance as function of optical trigger energy for 532 and $1064 \mathrm{~nm}$.

\subsection{PCSS DARK CURRENT}

The dark current through four of the PCSS devices was measured by a picoammeter while applying a dc voltage bias across the PCSS; picoammeter series combination. The voltage across the PCSS was monitored with a precision (1\%) $1000 \mathrm{M} \Omega$, resistive divider. The dark current varied linearly with the dc bias voltage for all four of the PCSS devices. The dark resistivity of the PCSS devices ranged from $10^{12}$ to $10^{13}$ $\Omega$-cm. Results for a typical dark current measurement is shown in Figure 6. The dark current for this PCSS ranged from a small fraction of a nanoampere up to $5.8 \mathrm{nA}$ at the highest dc bias of $1000 \mathrm{~V}$, as shown in Figure 6. The PCSS dark resistance and resistivity were calculated to be $170 \mathrm{G} \Omega$ and $3.2 \times 10^{12} \Omega-\mathrm{cm}$ for this PCSS. The ratio of the dark resistance to the minimum dynamic PCSS resistance of Figure $5(2 \Omega)$ is $8.5 \times 10^{10}$. The resistivity has been reduced by almost eleven orders of magnitude by exciting extrinsic levels in the bulk of the Vanadium compensated $6 \mathrm{H}-\mathrm{SiC}$. The dark current results are similar to previous measurements for semiinsulating $6 \mathrm{H}-\mathrm{SiC}[5]$.

\subsection{PCSS PHOTOCURRENTS}

The peak photocurrent in the PCSS has been measured as a function of bias voltage and optical pulse energy for both 532 and $1064 \mathrm{~nm}$ wavelengths. The peak photocurrent varies linearly with the bias voltage for fixed optical pulse energy as shown in Figure 7. The slope of the linear variation of peak

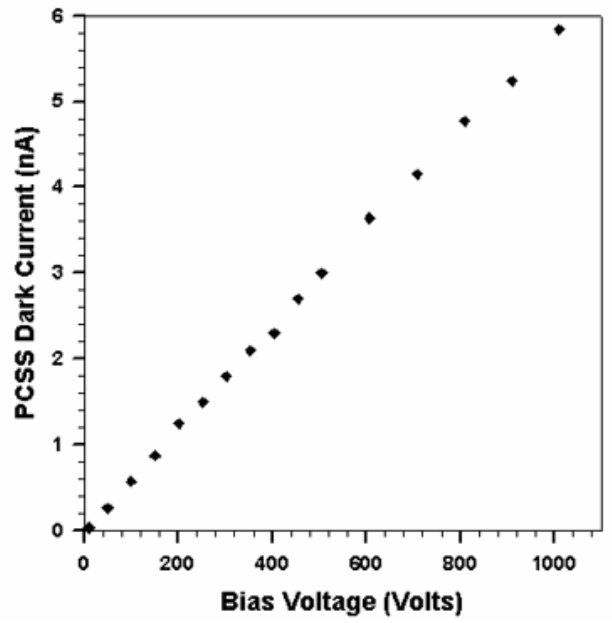

Figure 6. Variation of PCSS dark current with bias voltage.

photocurrent with bias voltage decreases with when the fixed optical pulse energy is reduced. This is what should be expected since we are optically modulating the PCSS resistance.

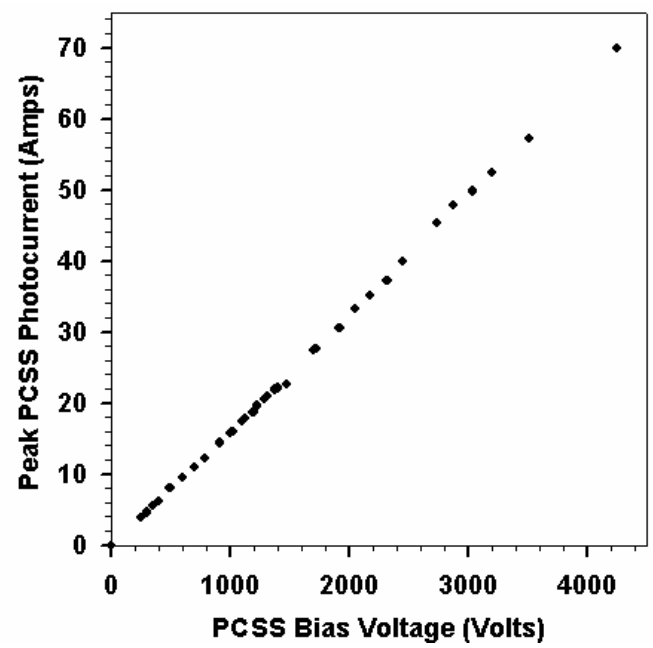

Figure 7. Peak PCSS photocurrent versus bias voltage for $13 \mathrm{~mJ}$ optical excitation at $1064 \mathrm{~nm}$.

The saturation of the peak PCSS photocurrent with increasing optical pulse energy for fixed bias voltage of 750 Volts is shown in Figure 8. The peak PCSS photocurrent for illumination with $532 \mathrm{~nm}$ was consistently 3- 4 A higher than the peak photocurrent obtained with $1064 \mathrm{~nm}$ illumination for this PCSS. This is a result of the $532 \mathrm{~nm}$ wavelength $(2.33$ $\mathrm{eV}$ ) exciting additional deep extrinsic levels compared to the 
$1064 \mathrm{~nm}$ wavelength $(1.17 \mathrm{eV})$. A consistent difference in peak PCSS photocurrent with excitation wavelength was observed in all the PCSS devices tested. However, the magnitude of the difference in peak PCSS photocurrent for different excitation wavelengths varied from 2 A up to 6 Amps. An explanation for the variation of the difference in peak photocurrents for 532 and $1064 \mathrm{~nm}$ excitation for different PCSS devices is that the impurity densities in the PCSS substrates vary. The substrates are cut from the a-plane of the $6 \mathrm{H}-\mathrm{SiC}$ crystal. The sections that were originally cut from the $6 \mathrm{H}-\mathrm{SiC}$ crystal measured $3.6 \mathrm{~cm}$ in the crystal growth (c) direction. The sections were then cut into $1.2 \mathrm{~cm}$ long substrates. We would expect variation in substrate impurity density along the growth direction because the impurity densities usually decrease during crystal growth. We expect somewhat different performance from substrate to substrate since we are exciting carriers from extrinsic (impurity) levels.

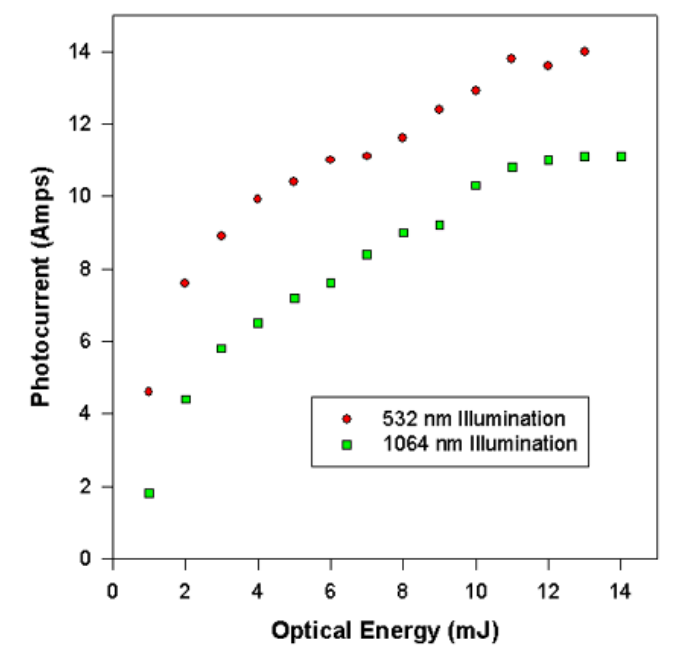

Figure 8. Peak PCSS photocurrent versus optical excitation energy for 750 Volt bias.

The nature of some of the different extrinsic levels being excited at the 1064 and $532 \mathrm{~nm}$ optical wavelengths is illustrated in Figure 9. The PCSS photocurrents for $11 \mathrm{~mJ}$ optical excitation at 532 and $1064 \mathrm{~nm}$ wavelengths show a significant difference in amplitude and pulse shape. The most notable difference is the long tail on the photocurrent excited at $532 \mathrm{~nm}$. The current tail persists and for one PCSS took approximately one millisecond to decay to zero. We believe crystal defects, that act as electron traps $[4,6]$ located 1.3-1.6 $\mathrm{eV}$ below the conduction band, are contributing the carriers in the photocurrent tail. Electrons are captured by the traps and thermally excited back into the conduction band resulting in a long decay time. The electron trap level has a recovery time of from a few to up to $200 \mu$ s. It also appears that the electron traps are filled prior to optical excitation based on the much faster rate of rise of photocurrent excited at $532 \mathrm{~nm}$. However, the electron trap level contributes only 2 of 15 A peak photocurrent at $532 \mathrm{~nm}$ excitation in Figure 9. We should expect that the electrons excited from vanadium acceptor level, that we believe constitutes the entire photocurrent at
$1064 \mathrm{~nm}$, will also be excited at $532 \mathrm{~nm}$. In addition, there appears to be at least a third component of photocurrent with a rapid rise and decay time that is present in the photocurrent at $532 \mathrm{~nm}$. We believe that the additional carriers are holes being excited into the valence band from vanadium acceptor levels that have not been ionized. A significant fraction of vanadium acceptor levels are not ionized because the Fermi level is pinned close to the vanadium acceptor level. In addition, Vanadium acceptor

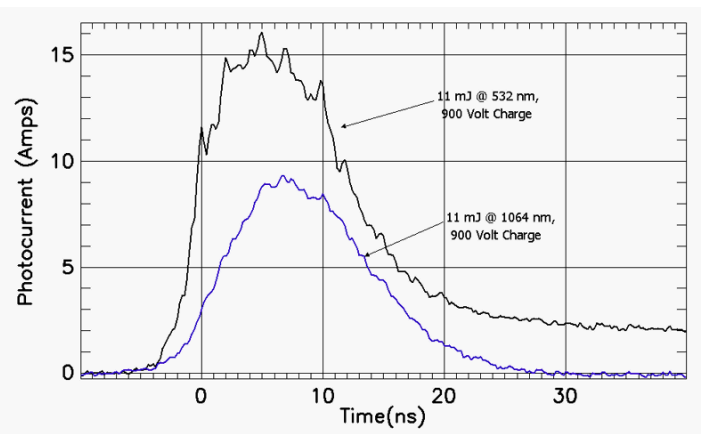

Figure 9. PCSS photocurrent temporal pulse for $11 \mathrm{~mJ}$ optical excitation at 532 and $1064 \mathrm{~nm}$ wavelengths.

levels are returned to the neutral state after electrons that they had captured are excited into the conduction band. Electrons can be excited from the valence band into the neutral vanadium acceptor levels that lie $2.3 \mathrm{eV}$ above the valence band by $532 \mathrm{~nm}(2.33 \mathrm{eV})$ wavelength light. Electrons that are excited into the neutral vanadium acceptor levels leave holes in the valence band that contribute to the conduction current of the PCSS.

We can estimate the triggering efficiency of the PCSS device from switching measurements at $13 \mathrm{~mJ}, 1064 \mathrm{~nm}$ optical excitation. We believe that the charge carriers are electrons excited from the vanadium acceptor level by the $1064 \mathrm{~nm}$ wavelength. The switch carrier density is given by:

$n(t)=\frac{l I_{s w}(t)}{q \mu a_{s w} V_{s w}(t)}$

where $V_{\mathrm{sw}}, \mathrm{I}_{\mathrm{sw}}, 1$, and $\mathrm{a}_{\mathrm{sw}}$ are the switch voltage, current, length, and area, $q$ is the elementary charge of an electron and $\mu$ is the electron mobility. Our switch length and area are 400 $\mu \mathrm{m}$ and $0.5 \mathrm{~cm}^{2}$, respectively. The electron mobility is estimated to be $200 \mathrm{~cm}^{2} / \mathrm{V}$-s. The peak carrier density is calculated using equation (1) from the minimum dynamic resistance $(10 \Omega)$ measurements. The peak number of electron carriers excited in the switch is calculated to be $2.5 \mathrm{e} 14 / \mathrm{cm}^{3}$ at $13 \mathrm{~mJ}$ excitation. The peak in carrier density corresponds to the peak optical intensity. The peak photon density injected into the switch volume is given by:

$n_{p h}=\frac{P_{p k} \tau_{r}}{h v L w d}$

where $\mathrm{P}_{\mathrm{pk}}, \tau_{\mathrm{r}}$, and $\mathrm{h} v$ are the peak optical power, electron recombination time and photon energy, respectively. L, w and 
$\mathrm{d}$ are the dimensions defining the switch volume irradiated by the optical pulse. The peak optical power is $1.625 \mathrm{e} 6 \mathrm{~W}$ for 13 $\mathrm{mJ}$ excitation at $1064 \mathrm{~nm}$. The electron recombination is estimated to be $\sim 2$ ns from our switching measurements. The switch volume irradiated by the optical pulse is $0.04 \mathrm{~cm}^{3}$. The peak photon density is calculated to be $4.35 \mathrm{e} 17 / \mathrm{cm}^{3}$. The ratio of peak photon to electron density is 1740 . One electron is excited into the conduction band for every 1740 photons injected into the switch volume. The triggering efficiency is 0.000575 , which is the inverse of the peak photon to electron ratio.

\subsection{PCSS ABSORPTION}

Optical transmission was measured at 532 and $1064 \mathrm{~nm}$ by measuring the optical energies incident on the front facet and exiting the rear facet of a PCSS device. The absorption coefficient was calculated from the transmission measurements assuming only linear absorption was occurring. Absorption coefficients of 1.29 and $1.59 \mathrm{~cm}^{-1}$ were calculated at $9 \mathrm{~mJ}$ applied optical energy for the 1064 and $532 \mathrm{~nm}$ wavelengths, respectively. One would expect an much larger absorption coefficient for $532 \mathrm{~nm}$ excitation compared to $1064 \mathrm{~nm}$ excitation, considering the minimum dynamic switch resistance is almost five times lower for $532 \mathrm{~nm}$ excitation at $9 \mathrm{~mJ}$ (see Figure 5). However, the absorption coefficient includes all optical loss mechanisms. The optical loss due to photon absorption at extrinsic levels may be small in comparison to other loss mechanisms since the impurity densities are approximately one part in a million of the bulk material. The dominant optical loss mechanisms could be scattering at the substrate surface and electrode metalization. In addition, the peak applied optical power density at $9 \mathrm{~mJ}$ excitation is $16 \mathrm{MW} / \mathrm{cm}^{2}$, which could be high enough to begin to produce nonlinear loss mechanisms.

\subsection{ELECTRICAL FAILURE OF PCSS}

A single PCSS device was tested to electrical failure of the $6 \mathrm{H}-\mathrm{SiC}$ substrate. The charge circuit was modified to produce a $3 \mu$ s rise time and the load resistor was reduced to $9 \mathrm{Ohms}$. The $1064 \mathrm{~nm}, 9 \mathrm{~mJ}$, optical pulse was applied after a $5 \mu \mathrm{s}$ flat top interval of the pulse bias voltage. The PCSS device was submerged in a transparent liquid dielectric to prevent tracking around the edge of the device. The PCSS suffered catastrophic failure after the sixth switching operation at a pulsed bias voltage of $11 \mathrm{kV}$. Failure was abrupt without any degradation in switching performance. Linear switch operation was observed at all charge voltages. No signs of nonlinear, avalanche, or "lock-on" [7] effects were observed. In fact, PCSS failure occurred after recovery from conduction at the $11 \mathrm{kV}$ bias voltage.

The $11 \mathrm{kV}$ bias produces an average electric field of 27.4 $\mathrm{MV} / \mathrm{m}$ in the $6 \mathrm{H}-\mathrm{SiC}$ substrate. Failure occurred through the bulk of the substrate directly under the edge of the device metalization, the location of greatest electric field enhancement. A peak field of $200 \mathrm{MV} / \mathrm{m}$ was numerically calculated for the failure point from the geometry of the failed device. We believe that making use of oxide coatings and $\mathrm{SiC}$ etching techniques will allow for PCSS device operations at significantly higher average electric fields.

\subsection{DIFFERENCES IN PCSS DEVICES}

The five PCSS devices fell into one of two categories based on minimum dynamic resistance. Four devices achieved a minimum dynamic on resistance of $10-11 \Omega$ at $13 \mathrm{~mJ}$ optical excitation at $1064 \mathrm{~nm}$. The remaining device had minimum dynamic on resistance of 2-3 $\Omega$ at optical excitation energies of $13 \mathrm{~mJ}$ at $1064 \mathrm{~nm}$. There was no visible difference in the switch facets. It was concluded that the difference in PCSS minimum dynamic resistance for the two switch categories was a result of differences in impurity densities. The density of the vanadium (V), nitrogen (N), boron (B), and aluminum (Al) impurities were measured using Secondary Ion Mass Spectroscopy (SIMS) for the "low" resistance switch and one of the four "high" resistance switches. Aluminum is an intentional p-type dopant in $6 \mathrm{H}-\mathrm{SiC}$. The $\mathrm{Al}$ density was an order of magnitude lower than the $\mathrm{N}, \mathrm{V}$ and $\mathrm{B}$ density and was assumed to have negligible effect on the switching process. The low resistance switch substrate had a higher density of $\mathrm{N}$ and lower density of $\mathrm{V}$ and $\mathrm{B}$ than the high resistance switch substrate. The lower resistance was attributed to the higher $\mathrm{N}$ density. The vanadium acceptor levels trap the electrons donated by the $\mathrm{N}$ levels. These are the electrons that are excited into the conduction band. The more electrons trapped by the vanadium acceptor levels, the lower the on resistance. The impurity density measurements have indicated a path toward optimizing the impurity levels in the vanadium compensated, $6 \mathrm{H}-\mathrm{SiC}$.

\section{CONCLUSION}

Bulk PCSS have been constructed from a-plane, vanadium compensated, semi-insulating $6 \mathrm{H}-\mathrm{SiC}$. The PCSS were switched on by optically exciting charge carriers from extrinsic levels using 532 and $1064 \mathrm{~nm}$ wavelength light. Operation in this manner allows for diffuse current conduction and the ability to take advantage of the high breakdown electric field of the bulk $6 \mathrm{H}-\mathrm{SiC}$ material. PCSS resistance was reduced from $170 \mathrm{G} \Omega$ to 2 and $11 \Omega$ by applying $13 \mathrm{~mJ}$ optical energy pulses at 532 and $1064 \mathrm{~nm}$ wavelengths, respectively. Photoconductivity measurements were performed to a bias voltage level of $4.25 \mathrm{kV}$ corresponding to an average electric of $10 \mathrm{MV} / \mathrm{m}$ between PCCS contacts. A slowly decaying current component with recombination times up to $200 \mu$ s were observed for $532 \mathrm{~nm}$ wavelength excitation. A single PCSS was tested to electrical failure at an average field of $27.4 \mathrm{MV} / \mathrm{m}$ in the $6 \mathrm{H}-\mathrm{SiC}$ substrate. The failure occurred at the point of highest electric field enhancement. We will utilize material processing techniques to achieve higher field operation of the PCSS devices. This initial work indicates that a compact, high voltage, high current switch can be developed from exciting the extrinsic levels in vanadium compensated, $6 \mathrm{H}-\mathrm{SiC}$ substrates. 


\section{ACKNOWLEDGEMENT}

This work was performed under the auspices of the U.S Department of Energy by the University of California, Lawrence Livermore National Laboratory under contract

W-7405-Eng-48.

\section{REFERENCES}

[1] S. Dogan, A. Teke, D. Huang, H. Morkoc, C. Roberts, J. Parish, B. Ganguly, M. Smith, R. Myers and S. Saddow, "4H-SiC photoconductive switching devices for use in high-power applications", Appl. Phys. Letts., Vol. 82, pp. 3107-3109, 2003.

[2] S. Sheng, M. Spencer, X. Tang, P. Zhou and G. Harris, "Polycrystalline cubic silicon carbide photoconductive switch", IEEE Electron Device Letts., Vol. 18, pp 372-374, 1997.

[3] K. Zhu, S. Dogan, Y. Moon, J. Leach, F. Yun, D. Johnstone, H. Morkoc, G. Li and B. Ganguly " Effect of $\mathrm{n}^{+}-\mathrm{GaN}$ subcontact layer on 4H-SiC high-power photoconductive switch", Appl. Phys. Letts. 86, p. 261108, 2005.

[4] M. Bickermann, D. Hofmann, T. Straubinger, R. Weingartner and A. Winnacker, "Preparation of semi-insulating Silicon Carbide by Vanadium doping during PVT bulk crystal growth", Materials Science Forum, Vol. 433-436, pp. 51-54, 2003.

[5] P. Cho, J. Goldhar, C. Lee, S. Saddow and P. Neudeck, "Photoconductive and photovoltaic response of high-dark-resistivity 6H-SiC devices", J. Appl. Phys., Vol. 77, pp. 1591-1599, 1995.

[6] T. Dalibor, G. Pensl, H. Matsunami, T. Kimoto, W. Choyke, A. Schoner and N. Nordell, "Deep defect centers in Silicon Carbide monitored with deep level transient spectroscopy", Physica Status Solidi (A) Appl. Research, Vol.162, pp. 199-225, 1997.

[7] G.Loubriel, M. O'Malley and F. Zutavern, "Toward pulsed power uses for photoconductive semiconductor switches: closing switches", $6^{\text {th }}$ IEEE Pulsed Power Conference, Arlington, VA, pp. 145-148, 1987.

James S. Sullivan received the B.E.E. degree from Manhattan College, Riverdale, NY and the M.S.E.E. degree from the State University of New York at Buffalo in 1977 and 1980, respectively. Since 1983 he has been an electrical engineer at the Lawrence Livermore National Laboratory, Livermore, CA. His research interests include optoelectronics, semiconductors and high voltage switching.

Joel R. Stanley received his AAS in Laser Electro-Optics from Texas State Technical College (TSTC), Waco, TX in 1995. He has been employed with Lawrence Livermore National Laboratory, Livermore, CA since 2001. His research interests include: laser optics and high voltage transmission line pulsers. 